\title{
CURRENT PERSPECTIVES ON THE MICROBIOTA-GUT-BRAIN AXIS IN CHRONIC NEURODEGENERATIVE DISORDERS
}

\author{
DIEGO ALBANI (*) e CARMEN GIORDANO (**)
}

Nota presentata dal m.e. Stefano Maiorana

(Adunanza del 29 giugno 2017)

SunTO. - Le patologie neurodegenerative croniche sono prevalenti a causa dell'invecchiamento della popolazione. Queste condizioni invalidanti includono la demenza (come la malattia di Alzheimer - AD) e i disturbi del movimento (come il morbo di Parkinson - PD). Negli ultimi decenni, abbiamo accumulato prove sperimentali sulle possibili cause molecolari di $\mathrm{AD} / \mathrm{PD}$, ma sono disponibili pochi farmaci approvati, sfortunatamente senza alcuna reale azione di modifica della malattia. Mentre i casi di $\mathrm{AD} / \mathrm{PD}$ aumentano costantemente nel mondo, nuove ipotesi sulla loro patogenesi sono attivamente sotto esame. Una è basata sull'asse microbiota-intestino-cervello, che postula un'influenza diretta o indiretta della comunità microbica dell'intestino umano sul sistema nervoso e le sue funzioni. Questa interazione bidirezionale può avere un ruolo anche nei disturbi neurodegenerativi, in questo campo la ricerca richiede modelli e strumenti per crescere e giungere all'applicazione clinica. A tale riguardo, i modelli innovativi di malattia in vitro stanno guadagnando attenzione, in particolare perché presentano caratteristiche desiderabili come condizioni di crescita tridimensionale (3D) e movimento dei fluidi interstiziali grazie alla microfluidica. In questa nota riassumiamo i concetti fondamentali del ruolo del microbiota intestinale nelle malattie come $\mathrm{AD} / \mathrm{PD}$, con particolare attenzione ai progetti di ricerca in corso sull'argomento che stanno cercando di risolvere quesiti clinici o elaborare soluzioni tecniche per la ricerca di base e le applicazioni industriali in questo settore.

(*) Department of Neuroscience, IRCCS - Istituto di Ricerche Farmacologiche Mario Negri, Milano, Italy.

(**) Department of Chemistry, Materials and Chemical Engineering "Giulio Natta”, Politecnico di Milano, Italy. E-mail: carmen.giordano@polimi.it 


\begin{abstract}
$* * *$
ABSTRACT. - Chronic neurodegenerative disorders are mostly due to population aging. These invalidating conditions include dementia (as Alzheimer's disease - AD) and movement disorders (as Parkinson's disease - PD). In the past decades, we have been accumulating experimental evidence on the molecular triggers of $\mathrm{AD} / \mathrm{PD}$ and few approved drugs are available, unfortunately with no real disease-modifying action. As $\mathrm{AD} / \mathrm{PD}$ cases constantly rise in the world, new hypothesis on their pathogenesis are actively under investigation. One of this is based on the microbiota-gut-brain axis, that postulates a direct or indirect influence of the microbial host community of human gut on the nervous system and its functions. This bidirectional interaction may play a role also in neurodegenerative disorders, even though research in this field requires models and tools for growing to a clinical translational perspective. To this respect, innovative in vitro engineered disease models are gaining attention, particularly because they feature desirable characteristics as tridimensional $(3 \mathrm{D})$ growing conditions and interstitial fluids movement recapitulation thanks to microfluidics. Here we summarize current data on the impact of gut microbiota on $\mathrm{AD} / \mathrm{PD}$ models and patients, with a focus on ongoing research projects on the topic that are trying to unravel clinical questions or elaborate technical solutions for research and industry applications in the field.
\end{abstract}

\title{
1. THE MICROBIOTA-GUT-BRAIN AXIS AND NEURODEGENERATIVE DISORDERS
}

\subsection{The gut microbiota}

The human gastrointestinal tract hosts many different microorganisms, mostly bacteria, but also fungi, yeasts, viruses, and archaea [1]. The bacterial populations are collectively defined "microbiota", and their whole genome "microbiome" [2].

It seems that the gut is colonized by trillions of bacteria belonging to hundreds of different species that are dynamic and different between individuals. Moreover, the microbiota can change during the course of life and can be affected by different factors such as diet, life style, diseases and assumption of drugs [3,4]. The most abundant phyla in adults are Bacteroidetes and Firmicutes that sum-up 80-90\% of the whole gut microbiota [1].

The microbiota in the gut has many functions important for human health. It is involved in modulation of inflammatory signaling and protection against infections, thanks to nutrient competition, leading to starvation of competing pathogens [5]. Another way is the pro- 
duction of inhibitory substances such as bacteriocins, that act as antimicrobial peptides. Intestinal microbiota allows also the fermentation of complex non-digestible carbohydrates and the synthetizes of Short Chain Fatty Acids (SCFAs), as acetate, propionate and butyrate [6]. The SCFAs have anti-inflammatory properties; maybe anti-cancer effects, and also contributes to the control of mucin production $[6,7]$.

The 'healthy gut microbiota' is very important also for human immune-system development and reactivity, thanks to the controlled exposition of the host to microbial strains [8]. Finally, the intestinal microbiota promotes the intestinal barrier homeostasis, keeping its thickness and stimulating the production of mucus layer and the expression of intestinal tight junctions.

\subsection{Microbiota-gut-brain-axis}

A connection between altered microbiota and changes in the central nervous system physiology are increasingly evident. Gut microbiota dysbiosis can increase the intestinal permeability, with translocation of endotoxins across the epithelial barrier and ensuing pro-inflammatory cytokines production [9]. The activation of enteric neurons and glial cells may result in neurological dysfunction spreading along the gutbrain-axis (for instance, by the vagus nerve) and might led to brain neurodegenerative disorders, such as Parkinson disease (PD) and Alzheimer disease $(\mathrm{AD})$. As a matter of fact, recent studies have found a reduced abundance of the Prevotellaceae bacteria family in PD patients compared with healthy controls, and an increased abundance of Enterobacteriaceae among those patients with the postural instability and gait difficulty phenotype compared to those with tremor-dominant PD [10].

In $\mathrm{AD}$, deposition of protein aggregates composed of amyloid- $\beta$ peptide and tau protein in CNS tissues compromises cognitive function, mainly memory. We have evidence that alterations of microbiota population by antibiotic treatment may affect learning and memory abilities [11], even if this association is hard to study with available models, mostly germ-free (GF) animal models [12], as they suffer from main limitations as the different composition of the animal's microbiota and the lack of a strict control on microbiota composition once inocu- 
lated. For this reason, future research in $\mathrm{AD} / \mathrm{PD}$ centered on microbiota's pathologic role would greatly benefit from innovative solutions. In this scenario, bidimensional (2D) cell culture systems are the traditional tools, however, the availability of state-of-the art, innovative three dimensional (3D) and engineered cell-based models will strongly improve in vitro cell model reliability.

In the following paragraphs, an highlight on traditional and innovative $3 \mathrm{D}$ and engineered cell models related to $\mathrm{AD} / \mathrm{PD}$ neurodegeneration will be provided, with a focus on an innovative multiorgan platform that, starting from state-of-the-art models, will represent the first in vitro model of the whole microbiota-gut-brain axis, useful to evaluate microbiota impact on brain cell pathophysiology.

\section{BIDIMENSIONAL AND TRIDIMENSIONAL CELL CULTURE SYSTEMS}

Cell culture is the process by which eukaryotic cells are grown outside their natural environment. After the cells of interest have been isolated from living tissue, typically a tumour, they can be maintained under controlled conditions, that vary from each cell type but generally consist of a plastic support with a liquid medium which supplies the essential nutrient and gasses, thus regulating the physio-chemical environment. Cell culture systems are indispensable tools for a wide range of basic and clinical in vitro research and can be classified between twodimensional (2D) and three-dimensional (3D) cultures.

Bidimensional (2D) culture has been used since the early 1900s, when Ross Granville Harrison established the methodology of tissue culture adapted from bacteriology. He maintained tissue explants outside the animal and observed the outgrowth of nerve cells over time [13]. The classical 2D culture is still a static dish culture, which mainly generates cell monolayers. Most 2D cultures are adhesion dependent and cannot be grown in suspension cultures without mechanical support, so cells are often put onto coated surfaces of flat plastic dishes.

Two-dimensional cultures, due to their simplicity, are still extensively employed in the majority of cell cultures. They are inexpensive, very well established and a rich body of literature exists to compare 
outcome measures. Moreover, 2D cultures are easy to control, observe, and analyze by imaging, and provide cells nutrients without any diffusional transport limitation [14].

On the other hand, they do not fully reflect the situation in vivo, where cells grow within a complex three-dimensional microenvironment, with vascular perfusion continuously supplying metabolites and removing catabolites. In addition, they exhibit increased drug sensitivity, due to their exposed surface, and generally show a reduced capability to rebalance when stimulated.

As a specific limitation for brain and its disease models, 2D neuronal networks show random connections with other types of neural cells and overlapping cellular responses, showing limitations in mimicking the $3 \mathrm{D}$ tissue specific characteristics.

Moreover, human neural cells cultured in flat, stiff, 2D environments typically display an irregular morphology and form unnatural cell-cell interactions. In this case, tissue specific architecture $(3 \mathrm{D}$ neuronal network), mechanical and biochemical cues and cell-cell communication are lost to various degrees. This can lead to physiological inaccuracies that can be extremely problematic for disease modelling and pre-clinical drug screening.

In addition, 2D cultures, even if derived from sophisticated cell models (e.g. with induced-pluripotent stem cells - iPSC) show reduced viability, neurite outgrowth and synaptic density [15].

The $2 \mathrm{D}$ condition, in AD/PD disease models, may have the additional important limitation of lacking a time-dependent extracellular/intracellular accumulation of aggregated proteins, probably as a result of a culture condition not favourable conditions to protein accumulation, as the proteins might diffuse in the relatively large volume of cell culture media and are likely to be removed during regular media changes, preventing aggregation [16].

\section{ENGINEERED MODELS OF NEURODEGENERATIVE DISORDERS}

Two key strategies are currently pursued to overcome 2D models also in neurodegneration: 3D culturing and microfluidics. Below we describe the most relevant $\mathrm{AD} / \mathrm{PD}$ disease models developed so far, 
focusing on engineered systems and devices employed that take advantage of the just described technologies.

\subsection{Alzheimer's disease models}

Alzheimer's disease is characterized by two pathological features: $A \beta$ plaques and neurofibrillary tangles, that are connected to AD's brain damage. In particular the amyloid hypothesis proposes that the accumulation of $\mathrm{A} \beta$ plaques within brain triggers AD's pathogenesis: it's necessary to develop in vitro AD's models to comprehend the complex mechanisms of AD's pathogenesis, and therefore to test therapeutic drugs. AD has been studied widely with patient-derived iPSCs: to overcome the observed 2D iPSC neuron cell culture models' inability to generate extracellular protein aggregations, the research has quickly passed to 3D and microfluidic systems.

The first 3D iPSC neuron culture model from familial AD patients (mutant APP and mutant presenilin) was developed by Choi and colleagues and it represented, for the first time, a disease model that displayed hallmarks of $\mathrm{AD}$ pathology: extracellular deposition of amyloid- $\beta$, including amyloid- $\beta$ plaques, and aggregates of phosphorylated tau, as well as filamentous tau [17]. The model was based on a human neural stem cell-derived 3D culture system from neural progenitor cells (hNPCs). Progenitor cells were differentiated into neuronal and glial cells as they were planted onto a BD Matrigel ${ }^{\mathrm{TM}}$ layer, used to provide a $3 \mathrm{D}$ support matrix and to prevent diffusion of $\mathrm{A} \beta$, allowing for high local concentrations sufficient to initiate aggregation [16].

More recent studies applied the 3D culture model to generate high throughput models for drug screening against tau aggregation [18], or to compare efficacy of drug candidates ( $\beta$-secretase or $\gamma$-secretase inhibitors) in 2D vs. 3D culture systems [19], as 3D culture models of $\mathrm{AD}$ are also relatively cheaper and faster than $\mathrm{AD}$ transgenic mouse model. In particular, Medda and colleagues used commercially available cell lines to generate iPSC-derived cortical neurons to reproduce a robust and scalable tau aggregation model for drug discovery. Also in this case, they used Matrige ${ }^{\mathrm{TM}}$ to have a thin-layer 3D culture [18].

The generation of brain organoids, initially developed to model human brain, has been used to study mechanisms of $\mathrm{AD}$, too. In fact, 
another way to reproduce $3 \mathrm{D}$ structures that can facilitate interstitial compartments of $A \beta$ deposition is cerebral organoids or neuro-spheroid, self-organizing structures of stem cells cultivated in vitro.

Lee and colleagues developed the first iPSC-differentiated 3D neuro-spheroid model derived from AD patients' blood. In this model iPSC derived from ante-mortem blood samples obtained from five AD patients were cultured on Geltrex ${ }^{\mathrm{TM}}$ matrix-coated plates [19]. The use of 3D spheroid involves a reduced diffusion of drugs and chronic dosing and may constitute a disadvantage for large-scale drug screening, because of the increased time requirement for uniform bioavailability within the $3 \mathrm{D}$ spheroids. However, there are many offsetted advantages of 3D environment: besides a better anatomic resemblance to mature brain, it expands 3D cells that reliably represents the native target of the drug, it also allows for microscopic evaluation of spatial features related to drug effects and it eventually permits to quantify drug levels that is not available in our traditional 2D assay system [19].

In the same period, Raja and colleagues reported that brain organoids from FAD patients recapitulate AD disease phenotypes and pathologies including amyloid aggregation, hyperphosphorylated tau, and endosome abnormalities, all of which were reduced by treatment with secretase inhibitors [20]. One very important aspect of this model is the spontaneous and time-dependent appearance of both amyloid and tau pathology, in agreement with the observations from the scaffolded 3D cultures of Choi and colleagues [16].

The above described 3D models lack controlled flow dynamics, circulating blood cells, and a brain-specific microenvironment and ECM. Several in vitro AD models have been developed in microfluidic systems to deal with some of the just reported limitations [21].

For instance, Yoon Jung Choi and colleagues studied the neurotoxicity of $A \beta$ oligomeric assemblies by developing a microfluidic system capable of generating a gradient of $A \beta$ oligomeric assemblies within microchannels. Neuron cells were directly exposed to $A \beta$ using a microfluidic culture system that could generate slow flow rates similar to those of interstitial flow in brain because of an osmotic pump into the platform [22]. The main result was the toxicity of oligomeric $A \beta$ aggregates, a well-documented feature of in vivo AD models [22].

Park and colleagues from the same group extended this work to 
develop a 3D AD model in a microfluidic system incorporating 3D neuro-spheres more closely mimicking the brain tissue [23], while tau propagation from neuron to neuron was examined within a microfluidic system composed of three distinct culture chambers connected by arrays of microgrooves [21]. This system examined uptake and propagation properties of different tau species derived from postmortem cortical extracts and brain interstitial fluid (ISF) of tau-transgenic mice, as well as human AD cortices [24].

\subsection{Parkinson's disease models}

Parkinson's disease symptoms result from the death of dopaminegenerating neurons in a deep-brain region called substantia nigra, a pathologic process possibly initiated by the abnormal accumulation of -synuclein protein in the form of Lewy bodies.

PD cellular models rely on different cell lines having similarities to dopaminergic neurons and susceptible to toxicity deriving from protein aggregates containing $\alpha$-synuclein.

Relevant lines are the neuroblastoma cell line SH-SY5Y and the pheochromocytoma cell line PC12, as well as Lund Human Mesencephalic cells (LUHMES) - which show a stable dopaminergic phenotype after differentiation protocols - and primary dopaminergic cultures from rat or mice [25]. In recent studies, similarly to $A D$, patient-derived iPSC have led to progresses taking a significant leap towards personalized medicine [26].

These cell models have been coupled to microfluidic systems to provide realistic in vivo-like models.

One of the first microfluidic platform virtually able to reproduce Parkinson's disease in vitro was developed by Lu et al. in order to study mitochondrial transport impairment as a contribute to PD neural degeneration. Lu et al. developed a microdevice with a large open chamber directly adjacent to an array of microchannels, which furthered axon isolation and orientation to better visualize the movement of mitochondria and detect their speed [27].

The microfluidic approach has been also important in models studying the proposed $\alpha$-synuclein spreading among cells as possible propagation system of PD pathology. 
For instance, Freundt et al. analysed the spreading of $\alpha$-synuclein neuron to neuron with the aid of microfluidic device, even if medium change was manual and not continuous. Their model employed cortical mouse neurons from E17 embryonic stage growing them directly in the microfluidic device in order to separate soma from axonal projection in fluidically isolated microenvironments. In this study, it was demonstrated that $\alpha$-synuclein fibrils are internalized, transported within axons, released, and subsequently taken up by additional neurons [28].

Finally, Fernandes et al. developed a microfluidic cell-culture platform for studying the communication between different cell populations, to allow in a dynamic environment the investigation of neurons/glia cell interactions. Of note, this dynamic platform avoids direct cell-cell contact, allowing cell to communicate either by diffusion or by perfusion of molecules [29].

\subsection{MINERVA project as flexible platform to model microbiota-gut-brain axis}

In the 2016, the European Research Council (ERC) Consolidator Grant Programme funded a technological project named "MIcrobiotaGut-BraiN EngineeRed platform to eVAluate intestinal microflora impact on brain functionality" (acronym: MINERVA, ERC-CoG-2016 - ID 724734), having the main aim to evaluate microbiota metabolism impact on brain functionality linked to neurodegeneration. In fact, MINERVA proposes an in vitro multi-organ-on-chip microfluidic platform, based on miniaturized, optically accessible devices, serially connected to recapitulate the microbiota-gut-brain axis main players. These organ-on-chips are the "Microbiota-compartment", hosting one or more bacterial strains relevant for human gut microbiota, the "Gutcompartment", featuring gut epithelial and immune system cells, and the most innovative "Brain-compartment", reproducing a physiologic blood-brain barrier model and two 3D human brain cell models, where neurons, astrocytes and microglia are cultured alone or in combination. It is clear that MINERVA platform tries, thanks to a multidisciplinary approach, to offer a substantial contribution to speed-up the research on microbiota-gut-brain axis, in particular for neurodegenerative disorders as Alzheimer's disease or Parkinson's disease. To get these results, 
it will develop the "Brain compartment" as a $3 \mathrm{D}$ in vitro disease model based on hydrogel scaffolds and human neural cells derived from iPSC, all features that, to a different extent, are currently already used in other available in vitro disease models that try to overcome limitations of standard 2D culturing techniques, as better detailed above.

\section{CONCLUSiOnS}

State-of-the-art on the role of gut microbiota in neurodegeneration is now facing the need of reliable research tools helping in solving the basic question of the existence of a causal link between microbiota composition or metabolic products and the molecular triggers of Alzheimer's or Parkinson's disease. Of course, this is would be a revolutionary hypothesis, as research in the field has been focused up to now almost exclusively on the brain and the central nervous system. The availability of innovative solutions (as the platform MINERVA is), based on 3D in vitro culturing conditions and microfluidics are a current hot-spot, as demonstrated by the recent work in the field referenced above, and they will become real laboratory-scale routine in a few years, with the related perspective of clinical translation of results. In fact, a major outcome of research done in this field will be the identification of dietary or pharmacologic approaches able to keep the microbiota in a healthy condition, with a forced step forward also for precision medicine, as the individual microbioma may be unique and varies over time, so requesting a personalized treatment in presence of a neurodegenerative disorder or, even better, for its prevention. Finally, the microbiota-gut-brain axis challenge is also the need of a truly multidisciplinarity involving neuroscience, immunology, gastroenterology, material science and bioengineering, a philosophy fully present in MINERVA and other recent technological solutions.

\section{ACKNOWLEDGEMENTS}

"MINERVA" project has received funding from the European Research Council (ERC) under the European Union's Horizon 2020 research and innovation programme (Grant Agreement No 724734)". 


\section{REFERENCES}

[1] Sekirov I, Russell SL, Antunes LC, Finlay BB. Gut microbiota in health and disease. Physiol Rev. 2010, 90:859-904.

[2] Pagliari D, Saviano A, Newton EE, Serricchio ML, Dal Lago AA, Gasbarrini A, Cianci R. Gut Microbiota-Immune System Crosstalk and Pancreatic Disorders. Mediators Inflamm. 2018, 2018:7946431.

[3] Clarke SF, Murphy EF, Nilaweera K, Ross PR, Shanahan F, O'Toole PW, Cotter PD. The gut microbiota and its relationship to diet and obesity: new insights. Gut Microbes. 2012, 3:186-202.

[4] Maukonen J, Saarela M. Human gut microbiota: does diet matter? Proc Nutr Soc. 2015, 74:23-36.

[5] Rolhion N, Chassaing B. When pathogenic bacteria meet the intestinal microbiota. Philos Trans R Soc Lond B Biol Sci. 2016, 371(1707). pii: 20150504. doi: 10.1098/rstb.2015.0504.

[6] Louis P, Flint HJ. Diversity, metabolism and microbial ecology of butyrate-producing bacteria from the human large intestine. FEMS Microbiol Lett. 2009, 294:1-8.

[7] Miquel S, Martín R, Rossi O, Bermúdez-Humarán LG, Chatel JM, Sokol H, Thomas M, Wells JM, Langella P. Faecalibacterium prausnitzii and human intestinal health. Curr Opin Microbiol. 2013, 16:255-61.

[8] Mazmanian SK, Liu CH, Tzianabos AO, Kasper DL. An immunomodulatory molecule of symbiotic bacteria directs maturation of the host immune system. Cell. 2005,122:107-18.

[9] Wang B, Yao M, Lv L, Ling Z, Li L. The Human Microbiota in Health and Disease. Engineering, 2017, 3: 71-82.

[10] Mulak A, Bonaz B. Brain-gut-microbiota axis in Parkinson's disease. World J Gastroenterol. 2015, 21:10609-20.

[11] Fung TC, Olson CA, Hsiao EY. Interactions between the microbiota, immune and nervous systems in health and disease. Nat Neurosci. 2017, 20:145-155.

[12] Martin CR, Osadchiy V, Kalani A, Mayer EA The Brain-Gut-Microbiome Axis. Cellular and Molecular Gastroenterology and Hepatology. 2018, 6: 133-148.

[13] Millet LJ, Gillette MU. Over a century of neuron culture: from the hanging drop to microfluidic devices. Yale J Biol Med. 2012, 85:501-521.

[14] Arber C, Lovejoy C, Wray S. Stem cell models of Alzheimer's disease: progress and challenges. Alzheimers Res Ther. 2017, 9:42.

[15] Siney EJ, Kurbatskaya K, Chatterjee S, Prasannan P, Mudher A, WillaimeMorawek S. Modelling Neurodegenerative Diseases In Vitro: Recent Advances in 3D IPSC Technologies AIMS Cell and Tissue Engineering. 2018, 2: 1-23.

[16] Choi SH, Kim YH, Hebisch M, Sliwinski C, Lee S, D’Avanzo C, Chen H, Hooli B, Asselin C, Muffat J, Klee JB, Zhang C, Wainger BJ, Peitz M, Kovacs DM, Woolf CJ, Wagner SL, Tanzi RE, Kim DY. A three-dimensional human neural cell culture model of Alzheimer's disease. Nature. 2014, 515:274-278.

[17] Ghaffari LT, Starr A, Nelson AT, Sattler R. Representing Diversity in the Dish: 
Using Patient-Derived in Vitro Models to Recreate the Heterogeneity of Neurological Disease. Front Neurosci. 2018,12:56.

[18] Medda X, Mertens L, Versweyveld S, Diels A, Barnham L, Bretteville A, Buist A, Verheyen A, Royaux I, Ebneth A, Cabrera-Socorro A. Development of a Scalable, High-Throughput-Compatible Assay to Detect Tau Aggregates Using iPSC-Derived Cortical Neurons Maintained in a Three-Dimensional Culture Format. J Biomol Screen. 2016 , 21:804-15.

[19] Lee HK, Velazquez Sanchez C, Chen M, Morin PJ, Wells JM, Hanlon EB, Xia W. Three Dimensional Human Neuro-Spheroid Model of Alzheimer's Disease Based on Differentiated Induced Pluripotent Stem Cells. PLoS One. 2016,11:e0163072.

[20] Raja WK, Mungenast AE, Lin YT, Ko T, Abdurrob F, Seo J, Tsai LH. SelfOrganizing 3D Human Neural Tissue Derived from Induced Pluripotent Stem Cells Recapitulate Alzheimer's Disease Phenotypes. PLoS One. 2016,11:e0161969.

[21] Osaki T, Shin Y, Sivathanu V, Campisi M, Kamm RD. In Vitro Microfluidic Models for Neurodegenerative Disorders. Adv Healthc Mater. 2018, 7

[22] Choi YJ, Chae S, Kim JH, Barald KF, Park JY, Lee SH. Neurotoxic amyloid beta oligomeric assemblies recreated in microfluidic platform with interstitial level of slow flow. Sci Rep. 2013, 3:1921.

[23] Park J, Lee BK, Jeong GS, Hyun JK, Lee CJ, Lee SH. Three-dimensional brainon-a-chip with an interstitial level of flow and its application as an in vitro model of Alzheimer's disease. Lab Chip. 2015,15:141-50.

[24] Takeda S, Wegmann S, Cho H, DeVos SL, Commins C, Roe AD, Nicholls SB, Carlson GA, Pitstick R, Nobuhara CK, Costantino I, Frosch MP, Müller DJ, Irimia D, Hyman BT. Neuronal uptake and propagation of a rare phosphorylated high-molecular-weight tau derived from Alzheimer's disease brain. Nat Commun. 2015, 6:8490.

[25] Lázaro DF, Pavlou MAS, Outeiro TF. Cellular models as tools for the study of the role of alpha-synuclein in Parkinson's disease. Exp Neurol. 2017, 298:162-171.

[26] Torrent R, De Angelis Rigotti F, Dell'Era P, Memo M, Raya A, Consiglio A. Using iPS Cells toward the Understanding of Parkinson's Disease. J Clin Med. 2015, 4:548-566.

[27] Lu X, Kim-Han JS, O'Malley KL, Sakiyama-Elbert SE. A microdevice platform for visualizing mitochondrial transport in aligned dopaminergic axons. J Neurosci Methods. 2012, 209:35-9.

[28] Freundt EC, Maynard N, Clancy EK, Roy S, Bousset L, Sourigues Y, Covert M, Melki R, Kirkegaard K, Brahic M. Neuron-to-neuron transmission of -synuclein fibrils through axonal transport. Ann Neurol. 2012, 72:517-24.

[29] Fernandes JT, Chutna O, Chu V, Conde JP, Outeiro TF. A Novel Microfluidic Cell Co-culture Platform for the Study of the Molecular Mechanisms of Parkinson's Disease and Other Synucleinopathies. Front Neurosci. 2016,10:511. 